\section{Arbeitszeugnis: Grenzen des Anspruchs}

Nach mehr als zwei Jahren müssen Ärzte ein Arbeitszeugnis nicht mehr nachbessern. Ein Korrekturanspruch ist dann "verwirkt", heißt es in einem Urteil des Hessischen Landesarbeitsgerichts (LAG). Im konkreten Fall hatte ein Arbeitnehmer sein Zeugnis erst zwei Jahre und acht Monate nach dem Ausscheiden aus der Firma gerügt - zu spät, so das LAG. Der Arbeitgeber habe davon ausgehen dürfen, dass der Angestellte mit dem Zeugnis einverstanden war. Ob die Änderungswünsche ursprünglich berechtigt gewesen wären, sei daher egal (18 Sa 602/12). Martin Wortmann

\section{Ärzte verharren weiter im Stimmungstief}

Ärzte, Zahnärzte und psychologische Psychotherapeuten beurteilen ihre aktuelle und die wirtschaftliche Lage der kommenden Monate wieder etwas optimistischer als noch vor einem halben Jahr. Der Medizinklimaindex (MKI) verbesserte sich zuletzt auf minus 6,4. Dies ist der zweitbeste Wert, den der MKI seit Start der Erhebung im Herbst 2006 je aufwies. Von Optimismus ist die Branche allerdings weit entfernt. Ärzte sind unter den für den MKI abgefragten Berufsgruppen nach wie vor die mit dem niedrigsten MKI (minus 9,2). Dirk Schnack

\section{Förderung exzellenter Medizinstudenten}

Unter dem Namen "Medical Excellence" fördert der auf Ärzte und andere Akademiker spezialisierte Finanzdienstleister MLP Human- und Zahnmedizinstudenten mit einem Stipendienprogramm. Die Manfred Lautenschläger-Stiftung unterstützt pro Jahr 15 angehende Mediziner mit $500 €$ pro Semester über maximal drei Jahre. Bewerben können sich Medizinstudenten, die an einer deutschen Universität eingeschrieben sind. Die Bewerbungsfrist läuft noch bis zum 31. Juli 2013. Infos zum Stipendienprogramm gibt es online unter www.medicalexcellence.de. Kerstin Mitternacht

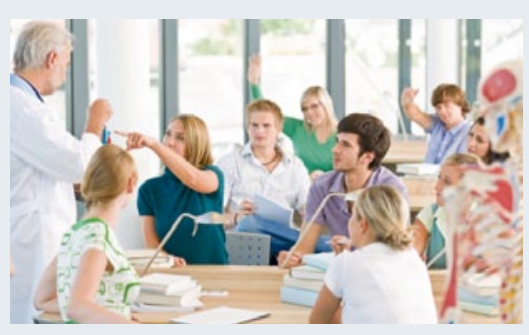

zum Thema Unabhängigkeit gegenüber Dritten. Außerdem müssen die Netze eine feste Managementstruktur nachweisen - dazu benötigen sie einen Geschäftsführer und einen ärztlichen Leiter oder Koordinator. Und da bei den förderungswürdigen Netzen „nicht der Gelderwerb im Fokus stehen darf“, wie Gibis erklärte, ist die Form der Aktiengesellschaft für Netze tabu.

\section{Dreistufiges Fördermodell}

Drei Versorgungsziele geben KBV und Krankenkassen in $\$ 4$ vor: die Patientenzentrierung, eine kooperative Berufsausübung und eine verbesserte Effizienz beziehungsweise Prozessoptimierung. An diesen Zielen und ihren Kriterien richten sich auch die drei Stufen der Förderung aus. Denn KBV und Krankenkassen wollen den Netzen durchaus die Chance geben, sich weiterzuentwickeln. Auch Netze, die weniger EDV und Standards einsetzen, aber trotzdem die regionale Versorgung nachhaltig verbessern oder sichern, sind nicht gänzlich von der Förderung ausgeschlossen.
Mindestens müssen Netze die Kriterien der Basisstufe erreichen. Und KBV und Krankenkassen haben durchaus sehr konkrete Vorstellungen, was Netze leisten müssen. Für die Grundförderung ist das allerdings mit nicht allzu großem Aufwand umsetzbar. Welcher Stufe welche Fördergelder zugesprochen werden, ist dann allerdings KV-Sache. Die KV muss auch entscheiden, ob sie überhaupt Fördergeld bereitstellt oder die Netze etwa unterstützt, an Add-on-Verträge mit den Krankenkassen zu kommen. Letzterer Weg wird voraussichtlich die Regel bei der Netzförderung sein, wie aus Anfragen bei KVen hervorgeht.

Aber nicht nur mit Geld werden Netze unterstützt. Die Rahmenvorgabe zeigt den Netzen auch andere Wege zu einer Unterstützung durch die KV. Etwa indem sie in $\$ 5$, Absatz 2, festschreibt, dass die KV Netzen spezifische Strukturdaten übermitteln muss. Das hilft in den Verhandlungen mit Krankenkassen.

RebekkaHöhl

Die Rahmenvorgabe im Internet: www.kbv.de//rechtsquellen/43483.html

\title{
Nachfolger muss ernsthaft an Fortführung der Praxis interessiert sein
}

\author{
In überversorgten Gebieten kann eine Nachfolgezulassung nur an Ärzte \\ gehen, die die Praxis tatsächlich fortführen wollen.
}

Ein in Arzt, der die Zulassung in eine Berufsausübungsgemeinschaft (BAG) einbringen und die Praxis als Angestellter fortführen will, scheidet als Nachfolger aus, wie das Bundessozialgericht (BSG) in Kassel entschied (B 6 KA 19/12 R). Der klagende Frauenarzt hatte sich 2007 um eine Praxisnachfolge in Kiel beworben und die Zulassung in eine Gemeinschaftspraxis eingebracht. Dort ist er als angestellter Arzt tätig. 2009 bewarb er sich erneut um eine Praxisnachfolge, diesmal im benachbarten Landkreis Plön. Den Sitz wollte er in eine BAG-Zweigpraxis einbringen. Den Zuschlag bekam aber eine Ärztin. Die dagegen gerichtete Klage des Gynäkologen war erfolglos.

Für die Nachfolge komme nur ein Arzt in Betracht, der die Praxis ,am bis- herigen Praxisort als Vertragsarzt" fortführt, so das BSG. Dies könne auch in einer überörtlichen BAG geschehen, nicht aber durch einen angestellten Arzt. „Nur so kann einer nicht gewollten Kommerzialisierung des Vertragsarztsitzes entgegengewirkt werden“, begründete das BSG. Die Praxisfortführung müsse vom Willen des Nachfolgers und dürfe nicht von dem des Arbeitgebers abhängen.

Zum Einfluss der Berufserfahrung auf die Nachfolgezulassung stellte das BSG klar, dass es auf das „Approbationsalter“ in der Regel nicht mehr ankommt, wenn konkurrierende Bewerber beide eine mehr als fünfährige Arzttätigkeit nach Abschluss der Weiterbildung vorweisen können.

Martin Wortmann 